\title{
An Optimal Algorithm for Computing the Largest Number of Red Nodes Daxin Zhu ${ }^{1, a}$, Xiaodong Wang $^{2, b,{ }^{*}}$ \\ ${ }^{1}$ Quanzhou Normal University, 362000 Quanzhou, Fujian, China. \\ ${ }^{2}$ Fujian University of Technology, Fuzhou, 350108 Fujian, China. \\ aemail: dex@qztc.edu.cn, bwangxd@139.com, ${ }^{*}$ Corresponding author
}

Keywords: Red-black trees; dynamic programming; data structure; time complexity

\begin{abstract}
In this paper, we investigate the problem to compute the largest number of red nodes in red-black trees in red-black trees. We first present a dynamic programming solution for computing $r(n)$, the largest number of red internal nodes in a red-black tree on $n$ keys in $O\left(n^{2} \log n\right)$ time. Then the algorithm is improved to a new $O(n)$ time algorithm. Based on the structure of the solution we finally present a linear time recursive algorithm using only $O(\log n)$ space.
\end{abstract}

\section{Introduction}

Red-black tree was invented in 1972 by Rudolf Bayer[2]. Guibas and Sedgewick named it red-black tree in 1978[4]. In their paper they studied the properties of red-black trees at length and introduced the red/black color convention. Andersson [1] gives a simpler-to-code variant of red-black trees. Weiss [7] calls this variant AA-trees. An AA-tree is similar to a red-black tree except that left children may never be red. In 2008, Sedgewick introduced a simpler version of the red-black tree called the left-leaning red-black tree[5] by eliminating a previously unspecified degree of freedom in the implementation. Red-black trees can be made isometric to either 2-3 trees or 2-4 trees,[5] for any sequence of operations.

The number of black nodes on any simple path from, but not including, a node $x$ down to a leaf is called the black-height of the node, denoted $b h(x)$. By the property (5), the notion of black-height is well defined, since all descending simple paths from the node have the same number of black nodes. The black-height of a red-black tree is defined to be the black-height of its root.

We are interested in the number of red nodes in red-black trees in this paper. We will investigate the problem that in a red-black tree on $n$ keys, what is the largest possible ratio of red internal nodes to black internal nodes, and what is the smallest possible ratio.

\section{Dynamic Programming Algorithm}

Let $T$ be a red-black tree on $n$ keys. The largest and the smallest number of red internal nodes in a red-black tree on $n$ keys can be denoted as $r(n)$ and $s(n)$ respectively. The values of $r(n)$ and $s(n)$ can be easily observed for the special case of $n=2^{k}-1$. In the general cases, we denote the largest number of red internal nodes in a subtree of size $i$ and black-height $j$ to be $a(i, j, 0)$ when its root red and $a(i, j, 1)$ when its root black respectively. Since in a red-black tree on $n$ keys we have $\frac{1}{2} \log n \leq j \leq 2 \log n$, we have,

$$
\gamma(n, k)=\max _{\frac{1}{2} \log n \leq j \leq 2 \log n} a(n, j, k)
$$

Furthermore, for any $1 \leq i \leq n, \frac{1}{2} \log i \leq j \leq 2 \log i$, we can denote, 


$$
\left\{\begin{array}{l}
\alpha_{1}(i, j)=\max _{0 \leq t \leq i / 2}\{a(t, j-1,1)+a(i-t-1, j-1,1)\} \\
\alpha_{2}(i, j)=\max _{0 \leq t \leq i / 2}\{a(t, j, 0)+a(i-t-1, j, 0)\} \\
\alpha_{3}(i, j)=\max _{0 \leq t \leq i / 2}\{a(t, j-1,1)+a(i-t-1, j, 0)\} \\
\alpha_{4}(i, j)=\max _{0 \leq t \leq i / 2}\{a(t, j, 0)+a(i-t-1, j-1,1)\}
\end{array}\right.
$$

\section{Theorem 1}

For each $1 \leq i \leq n, \frac{1}{2} \log i \leq j \leq 2 \log i$, the values of $a(i, j, 0)$ and $a(i, j, 1)$ can be computed by the following dynamic programming formula.

$$
\left\{\begin{array}{l}
a(i, j, 0)=1+\alpha_{1}(i, j) \\
a(i, j, 1)=\max \left\{\alpha_{1}(i, j), \alpha_{2}(i, j), \alpha_{3}(i, j), \alpha_{4}(i, j)\right\}
\end{array}\right.
$$

\section{Proof.}

For each $1 \leq i \leq n, \frac{1}{2} \log i \leq j \leq 2 \log i$, let $T(i, j, 0)$ be a red-black tree on $i$ keys and black-height $j$ with the largest number of red internal nodes, when its root red. $T(i, j, 1)$ can be defined similarly when its root black. The red internal nodes of $T(i, j, 0)$ and $T(i, j, 1)$ must be $a(i, j, 0)$ and $a(i, j, 1)$ respectively.

(1) We first look at $T(i, j, 0)$. Since its root is red, its two sons must be black, and thus the black-height of the corresponding subtrees $L$ and $R$ must be both $j-1$. For each $0 \leq t \leq i / 2$, subtrees $T(t, j-1,1)$ and $T(i-t-1, j-1,1)$ connected to a red node will be a red-black tree on $i$ keys and black-height $j$. Its number of red internal nodes must be $1+a(t, j-1,1)+a(i-t-1, j-1,1)$. In such trees, $T(i, j, 0)$ achieves the maximal number of red internal nodes. Therefore, we have,

$$
a(i, j, 0) \geq \max _{0 \leq t \leq i / 2}\{1+a(t, j-1,1)+a(i-t-1, j-1,1)\}
$$

On the other hand, we can assume the sizes of subtrees $L$ and $R$ are $t$ and $i-t-1$, $0 \leq t \leq i / 2$, WLOG. If we denote the number of red internal nodes in $L$ and $R$ to be $r(L)$ and $r(R)$, then we have that $r(L) \leq a(t, j-1,1)$ and $r(R) \leq a(i-t-1, j-1,1)$. Thus we have,

$$
a(i, j, 0) \leq 1+\max _{0 \leq t \leq i / 2}\{a(t, j-1,1)+a(i-t-1, j-1,1)\}
$$

Combining (4) and (5), we obtain,

$$
a(i, j, 0)=1+\max _{0 \leq t \leq i / 2}\{a(t, j-1,1)+a(i-t-1, j-1,1)\}
$$

(2) We now look at $T(i, j, 1)$. Since its root is black, there can be 4 cases of its two sons such as red and red, black and black, black and red or red and black. If the subtree $L$ or $R$ has a red root, then the black-height of the corresponding subtree must be $j$, otherwise, if its root is black, then the black-height of the subtree must be $j-1$.

In the first case, both of the subtrees $L$ and $R$ have a black root. For each $0 \leq t \leq i / 2$, subtrees $T(t, j-1,1)$ and $T(i-t-1, j-1,1)$ connected to a black node will be a red-black tree on $i$ keys and black-height $j$. Its number of red internal nodes must be $a(t, j-1,1)+a(i-t-1, j-1,1)$. In such trees, $T(i, j, 1)$ achieves the maximal number of red internal nodes. Therefore, we have, 


$$
a(i, j, 1) \geq \max _{0 \leq t \leq i / 2}\{a(t, j-1,1)+a(i-t-1, j-1,1)\}=\alpha_{1}(i, j)
$$

For the other three cases, we can conclude similarly that

Therefore, we have,

$$
\begin{gathered}
a(i, j, 1) \geq \max _{0 \leq t \leq i / 2}\{a(t, j, 0)+a(i-t-1, j, 0)\}=\alpha_{2}(i, j) \\
a(i, j, 1) \geq \max _{0 \leq t \leq i / 2}\{a(t, j-1,1)+a(i-t-1, j, 0)\}=\alpha_{3}(i, j) \\
a(i, j, 1) \geq \max _{0 \leq t \leq i / 2}\{a(t, j, 0)+a(i-t-1, j-1,1)\}=\alpha_{4}(i, j)
\end{gathered}
$$

$$
a(i, j, 1) \geq \max \left\{\alpha_{1}(i, j), \alpha_{2}(i, j), \alpha_{3}(i, j), \alpha_{4}(i, j)\right\}
$$

On the other hand, we can assume the sizes of subtrees $L$ and $R$ are $t$ and $i-t-1$, $0 \leq t \leq i / 2$, WLOG. In the first case, if we denote the number of red internal nodes in $L$ and $R$ to be $r(L)$ and $r(R)$, then we have that $r(L) \leq a(t, j-1,1)$ and $r(R) \leq a(i-t-1, j-1,1)$, and thus we have,

$$
a(i, j, 1) \leq \max _{0 \leq t \leq i / 2}\{a(t, j-1,1)+a(i-t-1, j-1,1)\}=\alpha_{1}(i, j)
$$

For the other three cases, we can conclude similarly that

Therefore, we have,

$$
\begin{aligned}
& a(i, j, 1) \leq \max _{0 \leq t \leq i / 2}\{a(t, j, 0)+a(i-t-1, j, 0)\}=\alpha_{2}(i, j) \\
& a(i, j, 1) \leq \max _{0 \leq t \leq i / 2}\{a(t, j-1,1)+a(i-t-1, j, 0)\}=\alpha_{3}(i, j) \\
& a(i, j, 1) \leq \max _{0 \leq t \leq i / 2}\{a(t, j, 0)+a(i-t-1, j-1,1)\}=\alpha_{4}(i, j)
\end{aligned}
$$

$$
a(i, j, 1) \leq \max \left\{\alpha_{1}(i, j), \alpha_{2}(i, j), \alpha_{3}(i, j), \alpha_{4}(i, j)\right\}
$$

Combining (11) and (16), we obtain,

$$
a(i, j, 1)=\max \left\{\alpha_{1}(i, j), \alpha_{2}(i, j), \alpha_{3}(i, j), \alpha_{4}(i, j)\right\}
$$

The proof is complete.

According to Theorem 1 , our algorithm for computing $a(i, j, k)$ is a standard 2-dimensional dynamic programming algorithm.

\section{Improvement of the Algorithm}

We have computed $r(n)$ and the corresponding red-black trees using Algorithm 1. Some pictures of the computed red-black trees with largest number of red nodes are listed. From these pictures of the red-black trees with largest number of red nodes in various size, we can observe some properties of $r(n)$ and the corresponding red-black trees as follows.

(1) The red-black tree on $n$ keys with $r(n)$ red nodes can be realized in a complete binary search tree, called a maximal red-black tree.

(2) In a maximal red-black tree, the colors of the nodes on the left spine are alternatively red, black, $\cdots$, from the bottom to the top, and thus the black-height of the red-black tree must be $\frac{1}{2} \log n$.

(3) In a maximal red-black tree of $k$ levels, if all of the nodes of the last two levels $(k, k-1)$ and all of the black nodes of the last third level $(k-2)$ are removed, the remaining tree is also a maximal red-black tree.

From these observations, we can improve the dynamic programming formula of Theorem 1 further. The first improvement can be made by the observation (2). Since the black-height of the 
maximal red-black tree on $i$ keys must be $\frac{1}{2} \log i$, the loop bodies of the Algorithm 1 for $j$ can be restricted to $j=\frac{1}{2} \log i$ to $1+\frac{1}{2} \log i$, and thus the time complexity of the dynamic programming algorithm can be reduced immediately to $O\left(n^{2}\right)$. By the observation (3), the time complexity of the algorithm can be reduced substantially as follows.

\section{Theorem 2}

Let $n$ be the number of keys in a red-black tree, and $r(n)$ be the largest number of red nodes in a red-black tree on $n$ keys. The values of $r(n)$ can be computed by the following recursive formula.

$$
r(n)= \begin{cases}n-\lfloor\log n\rfloor & n<8 \\ r(p)+q & n \geq 8\end{cases}
$$

where

$$
\left\{\begin{array}{l}
p=2^{\lfloor\log n\rfloor-2}+\left\lceil\left(n-2^{\lfloor\log n\rfloor}+1\right) / 4\right\rceil-1 \\
q=n-2^{\lfloor\log n\rfloor-1}-2\left\lceil\left(n-2^{\lfloor\log n\rfloor}+1\right) / 4\right\rceil+1
\end{array}\right.
$$

\section{Proof.}

Let $T$ be a maximal red-black tree of size $n$. It is obvious that $T$ has $k=1+\lfloor\log n\rfloor$ levels.

(1) The formula can be verified directly for the case of $n<8$.

(2) In the case of $n \geq 8$, we have $k>3$. The number of nodes in the last level of $T$ must be $s=n-2^{\lfloor\log n\rfloor}+1$. These nodes are all red nodes of $T$. It is readily seen that every 4 red nodes in the last level correspond to 2 black nodes in level $k-1$ of $T$. Thus the number of black nodes in level $k-1$ must be $b=2\left\lceil\left(n-2^{\lfloor\log n\rfloor}+1\right) / 4\right\rceil$. It follows that the number of red nodes in level $k-1$ of $T$ is $2^{\lfloor\log n\rfloor-1}-b$. Therefore, the number of red nodes in the last two levels of $T$ is $s+2^{\lfloor\log n\rfloor-1}-b$, which is exactly $q=n-2^{\lfloor\log n\rfloor-1}-2\left\lceil\left(n-2^{\lfloor\log n\rfloor}+1\right) / 4\right\rceil+1$.

Let $T^{\prime}$ be the subtree of $T$ by removing all of the nodes of the last two levels $(k, k-1)$ and all of the black nodes in level $k-2$ from $T$. Since every 2 black nodes in level $k-1$ correspond to 1 red node in level $k-2$ of $T$, the number of red nodes in level $k-2$ is obviously $b / 2$, and thus the size of $T^{\prime}$ must be $2^{\lfloor\log n\rfloor-2}+b / 2$, which is exactly $p=2^{\lfloor\log n\rfloor-2}+\left\lceil\left(n-2^{\lfloor\log n\rfloor}+1\right) / 4\right\rceil-1$. It follows from observation (3) that $r(n)=r(p)+q$.

The proof is complete.

According to Theorem 2, a new recursive algorithm for computing the largest number of red internal nodes in a red-black tree on $n$ keys can be implemented.

For the same problem, we can build another efficient algorithm in a different point of view. Let us look at the sequence of the values of $r(n)$ listed in the increasing order of $n$.

If we list the sequence as a triangle $t(i, j), i=0,1, \cdots, j=1,2, \cdots, 2^{i}$, then we can observe some interesting structural properties of $r(n)$.

It is readily seen that the values in each row have some regular patterns as follows.

(1) For the fist elements $t(i, 1)$ in each row $i=0,1, \cdots$, we have,

$t(2 j+1,1)=2 t(2 j, 1)+1, t(2 j, 1)=2 t(2 j-1,1), j=1,2, \cdots$.

(2) For the elements $t\left(i, 2^{i-1}+1\right)$ in each row $i=1,2, \cdots$, we have, $t\left(i, 2^{i-1}+1\right)=2^{i}-1$. 
(3) For the elements $t(i, j), 2 \leq j \leq 2^{i-1}$ in each row $i=2,3, \cdots$, we have, $t(i, j)=t(i-1, j)+c$ where $c$ is a constant.

(4) For the elements $t(i, j), 2^{i-1}+2 \leq j \leq 2^{i}$ in each row $i=2,3, \cdots$, we have, $t(i, j)=t\left(i-1, j-2^{i-1}\right)+d$ where $d$ is a constant.

In the insight of these observations, we can build another efficient algorithm to compute $r(n)$ as follows.

\section{Theorem 3}

Let $n$ be the number of keys in a red-black tree, and $r(n)$ be the largest number of red nodes in a red-black tree on $n$ keys. If the values of $r(n)$ are listed as a triangle $t(i, j), i=0,1, \cdots, j=1,2, \cdots, 2^{i}$ as shown in Table 2 , then the values of $t(i, j)$ can be computed by the following recursive formula.

$$
t(i, j)= \begin{cases}i & i<2 \\ \xi(i) & 2 \leq i, j=1 \\ t(i-1, j)+\xi(i-1) & 2 \leq i, 2 \leq j \leq 2^{i-1} \\ 2^{i}-1 & 2 \leq i, j=2^{i-1}+1 \\ t\left(i-1, j-2^{i-1}\right)+\eta(i) & 2 \leq i, 2^{i-1}+2 \leq j \leq 2^{i}\end{cases}
$$

where

$$
\left\{\begin{array}{l}
\xi(i)=\frac{2}{3}\left(2^{i}-\frac{3+(-1)^{i}}{4}\right)=\left\lceil\frac{2}{3}\left(2^{i}-1\right)\right\rceil \\
\eta(i)=\frac{1}{3}\left(2^{i+1}+(-1)^{i}\right)=\left\lfloor\frac{1}{3}\left(2^{i+1}+1\right)\right\rfloor
\end{array}\right.
$$

According to Theorem 3, a recursive algorithm for computing the values of $t(i, j)$ can be implemented as the following Algorithm.

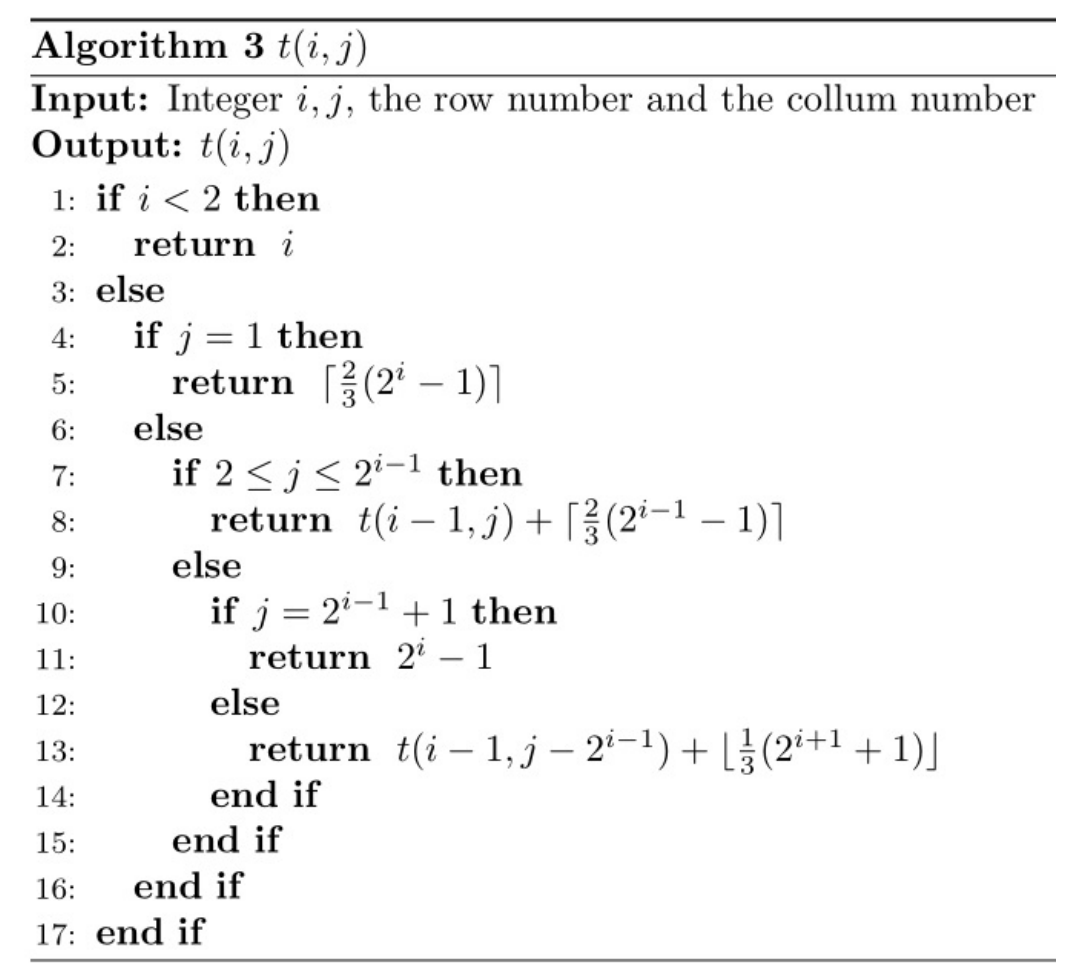


It can be seen that for any positive integer $n$, if $t(i, j)=r(n)$, then $i=\lfloor\log (n+1)\rfloor$ and $j=n-2^{\lfloor\log (n+1)\rfloor}+2$. In a call of Algorithm, $t\left(\lfloor\log (n+1)\rfloor, n-2^{\lfloor\log (n+1)\rfloor}+2\right)$ will return the value of $r(n)$. It is obvious that the recursive depth of the Algorithm is at most $\lfloor\log (n+1)\rfloor$. Therefore, our new algorithm requires only $O(\log n)$ time.

\section{Acknowledgement}

This work was supported in part by the Natural Science Foundation of Fujian (Grant No.2013J01247), Fujian Provincial Key Laboratory of Data-Intensive Computing and Fujian University Laboratory of Intelligent Computing and Information Processing.

\section{References}

[1] Andersson, Balanced search treesmade simple, In Proceedings of the Third Workshop on Algorithms and Data Structures, vol. 709 of Lecture Notes in Computer Science, 1993, pp. 60-71.

[2] R. Bayer, Symmetric binary B-trees: Data structure and maintenance algorithms, Acta Informatica, 1(4), 1972, pp. 290-306.

[3] Cormen T.H., Leiserson C.E., Rivest R.L., Stein C., Introduction to algorithms, 3rd ed., MIT Press, Cambridge, MA, 2009.

[4] Leo J. Guibas and Robert Sedgewick, A dichromatic framework for balanced trees, In Proceedings of the 19th Annual Symposium on Foundations of Computer Science, 1978, pp. 8-21.

[5] Robert Sedgewick, Left-leaning Red?CBlack Trees, http://www.cs.princeton.edu/ rs/talks/ LLRB/LLRB.pdf

[6] Henry S. Warren, Hacker's Delight, Addison-Wesley, second edition, 2002.

[7] Mark Allen Weiss, Data Structures and Problem Solving Using C++, Addison-Wesley, second edition, 2000. 